\title{
A Simple Method to Isolate and Expand Human Umbilical Cord Derived Mesenchymal Stem Cells: Using Explant Method and Umbilical Cord Blood Serum
}

\author{
Ghmkin Hassan $^{1}$, Issam Kasem ${ }^{2,3}$, Chadi Soukkarieh ${ }^{2,3}$, Majd Aljamali ${ }^{1,3}$ \\ ${ }^{1}$ Department of Microbiology and Biochemistry, Faculty of Pharmacy, Damascus University, Damascus, Syria \\ ${ }^{2}$ Department of Animal Biology, Faculty of Sciences, Damascus University, Damascus, Syria \\ ${ }^{3}$ National Commission for Biotechnology (NCBT), Damascus, Syria
}

Background and Objectives: Mesenchymal stem cells (MSCs) are multipotent stem cells that can be isolated from umbilical cords and are therapeutically used because of their ability to differentiate into various types of cells, in addition to their immunosuppressive and anti-inflammatory properties. Fetal bovine serum (FBS), considered as the standard additive when isolating and culturing MSCs, has a major limitation related to its animal origin. Here, we employed a simple and economically efficient protocol to isolate MSCs from human umbilical cord tissues without using digestive enzymes and replacing FBS with umbilical cord blood serum (CBS).

Methods and Results: MSCs were isolated by culturing umbilical cord pieces in CBS or FBS supplemented media. Expansion and proliferation kinetics of cells isolated by explant method in the presence of either FBS or CBS were measured, with morphology and multi-differentiation potential of expanded cells characterized by flow cytometry, RT-PCR, and immunofluorescence. MSCs maintained morphology, immunophenotyping, multi-differentiation potential, and self-renewal ability, with better proliferation rates for cells cultured in CBS compared to FBS supplement media.

Conclusions: We here present a simple, reliable and efficient method to isolate MSCs from umbilical cord tissues, where cells maintained proliferation, differentiation potential and immunophenotyping properties and could be efficiently expanded for clinical applications.

Keywords: Mesenchymal stem cells, Cord blood serum, Isolation, Differentiation

\footnotetext{
Accepted for publication July 4, 2017, Published online November 30, 2017

Correspondence to Ghmkin Hassan

Department of Microbiology and Biochemistry, Faculty of Pharmacy, Damascus University, PO Box 10769, Damascus, Syria

Tel: +963112331919, Fax: +963112331919

E-mail: hsn.ghmkin@gmail.com

Co-Correspondence to Majd Aljamali

Department of Biochemistry and Microbiology, Faculty of Pharmacy, Damascus University, Damascus, Syria

Tel: +963115138306, Fax: +963115130104

E-mail: maljamali@gmail.com

(c) This is an open-access article distributed under the terms of the Creative Commons Attribution Non-Commercial License (http://creativecommons.org/licenses/by-nc/4.0/), which permits unrestricted non-commercial use, distribution, and reproduction in any medium, provided the original work is properly cited.

Copyright (c) 2017 by the Korean Society for Stem Cells Research
} 


\section{Introduction}

Mesenchymal stem cells (MSCs) are multipotent stem cells with main potential to differentiate into adipocytes, osteoblasts, chondrocytes $(1,2)$, and possible differentiation potential into hepatocytes, cardiomyocytes, skeletal myocytes and neurons (3). Recently, MSCs have been increasingly used in regenerative medicine because of their ability to treat tissue injury $(4,5)$, and their immunomodulatory properties (6-8); therefore, they have been part of clinical practice for more than 10 years (9) and were successfully isolated from a number of tissues including: bone marrow (10), adipose tissue $(11,12)$, umbilical cord blood (13), placenta (14), amniotic fluid $(15,16)$ in addition to umbilical cords (1). Umbilical cord tissue is one of the major sources of MSCs since isolation of umbilical cord MSCs (UCMSCs) is easier, does not require an invasive procedure and has a higher proliferation potential compared with other sources (2). Moreover, umbilical cord is usually discarded after birth so the collection of cells does not raise any major ethical concerns (17).

In standard culture conditions, MSCs are adherent to plastic surfaces of culture vessels and could be functionally isolated from tissues based on this ability. MSCs are also defined by their multi-differentiation potency and expression of cell surface markers (CD105, CD73, and CD90) and lack expression of (CD45, CD34, CD14) (18, 19). There are two main techniques to isolate MSCs from umbilical cord tissue: enzyme digestion and explants (2). Digestion-based protocols generally use enzymes like collagenase and hyaluronidase to digest tissues and release cells from the extracellular matrix of Wharton jelly, which then adhere to the surface of culture vessels (19). The main disadvantage of these protocols is that enzyme solutions are often poorly defined and have a broad range of incubation times (30 min 16 hours) depending on the purity and source of the enzymes. This raises the risk of degradation of cellular external lamina preventing MSCs from adhering to the surface of culture vessels after enzymatic digestion $(2,20)$.

On the other hand, explant protocols are cost-effective and simpler than enzymatic digestion protocols since they don't require incubation with enzymes and depend on the MSCs ability to migrate from umbilical cord tissues and adhere to the surface of culture vessels (21). Explant protocols differ from each other by a number of factors that could affect the quality of isolation protocol such as: size of explants (22), type of culture vessels (plates, petri dishes or flasks), supplements added to culture media (21), and time required to change medium for first time
(22-24). In this regard, many laboratories develop and optimize their own isolation and expansion protocol $(20,25)$.

Almost all protocols use fetal bovine serum (FBS) as a supplement in culture media as a source of hormones, attachment factors and other molecules required for isolation and expansion of MSCs in both research and clinical fields. However, FBS supplement medium has some limitations associated with clinical applications due to its animal source. These limitations include contamination risk by prions, bacteria, and viruses, and the potential for immunological reactions against xeno-genic components, in addition to high lot-to- lot variations $(26,27)$. In recent years, xenogenic -free and serum-free supplements such as human serum $(28,29)$, platelets lysate (27), cord blood serum (CBS) (30) and chemical defined media have been tested as FBS alternatives (31). Although, Serum-free media eliminate batch-to-batch variability they may still contain xenogenic proteins and has risk to transmitting infections. In addition, serum-free media are highly expensive compared with human blood derived supplement media; serum, plasma and platelet lysates (32).

CBS contains cytokines and growth factors which are required for proliferation and growth of stem cells such as (EGF, FGF, NGF, VEGF, PDGF, IGF, TGF, interleukins, interferons). It has been used as FBS substitute in culture media for animal MSCs, human bone-marrow- MSCs and umbilical cord blood derived MSCs (30, 33).

Optimization of simple, cost-effective and xenogenicfree protocols for isolating and expanding UCMSCs is an important factor in the successful application to deliver a sufficient number of cells to patients (20). Therefore, this study investigated the suitability of using pooled CBS as a replacement for FBS for developing suitable protocol to isolate UCMSCs by explant method without using digesting enzymes and in the expansion of UCMSCs.

\section{Materials and Methods}

\section{Preparation of pooled CBS}

After receiving the approval from Damascus University ethical committee, written informed consents were obtained from all participating pregnant women for donating their cord tissues and blood. Cord blood was collected from six donors after delivery using $50 \mathrm{ml}$ sterile canonical tube without anti-coagulation. Blood then was allowed to clot for $2 \sim 4 \mathrm{~h}$ at room temperature. The clotted blood was centrifuged at $1,000 \mathrm{~g}$ at $20^{\circ} \mathrm{C}$ for $30 \mathrm{~min}$. Sera were collected and pooled together to eliminate variations, then inactivated by heating at $56^{\circ} \mathrm{C}$ for $30 \mathrm{~min}$. Finally, pooled cord blood serum was filtrated using $0.22-\mu \mathrm{m}$ filters 
(Ministart, Sartourius Stediumbiotech, Germany), and was stored at $-80^{\circ} \mathrm{C}$.

\section{Isolation of mesenchymal stem cell from umbilical cord tissue}

Three human umbilical cords were obtained from women with healthy pregnancies during caesarean deliveries at the end of gestation after signing informed consents. Umbilical cords (UCs) were collected in phosphate-buffered saline (PBS) (Euroclone, Italy) supplemented with antibiotics, $100 \mathrm{U} / \mathrm{ml}$ penicillin and $100 \mu \mathrm{g} / \mathrm{ml}$ streptomycin (Euroclone, Italy) and transported to the laboratory. UCs were washed with PBS under a sterile laminar flow cell culture hood and cut into $5 \mathrm{~cm}^{2}$ segments. The segments were cut longitudinally, and blood vessels were removed, and segments transferred to $25 \mathrm{~cm}^{2}$ flasks and incubated at $37^{\circ} \mathrm{C}$ in a humidified atmosphere with $5 \% \mathrm{CO}_{2}$ in DMEM (Euroclone, Italy) supplemented with either 10\% FBS (Euroclone, Italy) or 10\% CBS. Flasks were left undisturbed for 7 days, after which the medium was changed for the first time and later was changed every $3 \sim 4$ days. After 2 weeks, the UC explants were removed and the adherent cells were allowed to expand until they reach about $80 \%$ confluence. Morphology of isolated and expanded MSC cells was assessed using inverted microscope IX50 (Olympus, Japan) during the cell culture period.

\section{Expansion and Proliferation kinetics}

After reaching about $80 \%$ confluence, cells were subcultured using trypsin, EDTA $0.05 \%$ (Euroclone, Italy) to detach cells, then were centrifuged at $200 \mathrm{~g}$ for $5 \mathrm{~min}$. Cells were counted using trypan blue exclusion and reseeded at 10,000/well density in 12 well plate in DMEM supplemented with either $10 \%$ FBS or $10 \%$ CBS. After 7 days, cells were trypsinized and counted. This procedure was repeated from passage 1 to passage 3. Population doubling time (PDT) was calculated for passage 1 and passage 3 according to the following equation:

$$
\mathrm{PDT}=\triangle \mathrm{T} \times\left(\log \mathrm{N}_{\mathrm{H}}-\log \mathrm{N}_{0}\right) / \log (2)
$$

where, $\Delta \mathrm{T}$ : is culture period in hours, $\mathrm{N}_{\mathrm{H}}$ : number of viable cells at the end of passage and $\mathrm{N}_{0}$ : number of plated cell.

The fold increase was also calculated according to the equation:

Fold increase $=\mathrm{N}_{\mathrm{H}} / \mathrm{N}_{0}$

\section{In vitro differentiation}

Adipogenic and osteogenic differentiation: At the end of P2, cells were plated at 5,000/well in 96 well plate in (DMEM $+10 \% \mathrm{CBS})$ or (DMEM $+10 \% \mathrm{FBS})$ medium. After reaching 80 100\% confluence, adipogenic differentiation medium (hMSC adipogenic differentiation kit, Euroclone, Italy) or osteogenic differentiation medium (hMSC osteogenic differentiation kit, Euroclone, Italy) were added, and media were changed every $3 \sim 4$ days. After 3 weeks, cells were fixed with $10 \%$ formaldehyde. For adipogenic differentiation, lipid formation was assessed by staining with $2 \%$ sudan III for $5 \mathrm{~min}$ at room temperature then cells were washed with distilled water. For osteogenic differentiation, calcium deposits were detected by staining with $2 \%$ alizarin red $(\mathrm{pH} 4.2)$ for 20 min at room temperature followed by washing with distilled water. Lipid droplets and calcium deposits were detected by inverted microscope IX50 (Olympus, Japan). Control cells were maintained in (DMEM $+10 \% \mathrm{CBS})$ or (DMEM+10\% FBS).

\section{Characterization of MSC}

Molecular phenotyping: Total RNA was extracted from cells at end of P3 using (RNeasy Mini Kit-Qiagen, Germany). Reverse transcription was carried out using (RevertAid First Strand Synthesis Kit-Thermo fischer, USA) and RT-PCR reaction was performed using (Dream Taq, Thermo fischer, USA) to detect expression of CD44, CD90, CD105, using beta-actin gene expression as a control under the condition detailed in Table 1.

Primers that were used in RT-PCR reaction are shown in the Table 2.

$2 \%$ agarose gel electrophoresis was performed to detect PCR products using $100 \mathrm{pb}$ ladder (GeneDireX, Taiwan) and ethidium bromide (Carl Roth, Germany) to visualize amplicons.

Immunophenotypic characterization: Cells at passage 2 , isolated and expended in medium supplemented with

Table 1. PCR conditions

\begin{tabular}{lcc}
\hline \multicolumn{1}{c}{ Step } & Temperature & Time \\
\hline Initial Denaturation & $95^{\circ} \mathrm{C}$ & $5 \mathrm{~min}$ \\
Cycle & & \\
Denaturation & $95^{\circ} \mathrm{C}$ & $30 \mathrm{~s}$ \\
Annealing & $*$ Determined according to target & $30 \mathrm{~s}$ \\
Extension & $72^{\circ} \mathrm{C}$ & $30 \mathrm{~s}$ \\
Final Extension & $72^{\circ} \mathrm{C}$ & $5 \mathrm{~min}$ \\
\hline
\end{tabular}

*Annealing temperatures: $\mathrm{CD} 44: 53^{\circ} \mathrm{C}, \mathrm{CD} 90: 54^{\circ} \mathrm{C}, \mathrm{CD} 105: 55^{\circ} \mathrm{C}$, Beta-actin: $53^{\circ} \mathrm{C}$. 
Table 2. Primers used in RT-PCR reaction

\begin{tabular}{ccc}
\hline Gene & PCBI accession number & Primer sequence \\
\hline CD44 & NM_000610 & 5-AAGGTGGAGCAAACACAACC-3 \\
CD90 & 5M_006288 & 5-GGACTGAGATCCCAGAACCA-3 \\
& & 5-ACGAAGGCTCTGGTCCACTA-3 \\
CD105 & NM_000118 & 5-CACTAGCCAGGTCTCGAAGG-3 \\
& NM_001101 & 5-CTGAGGACCAGAAGCACCTC-3 \\
Beta-actin & 5-GATGAGATTGGCATGGCTTT-3 \\
\end{tabular}
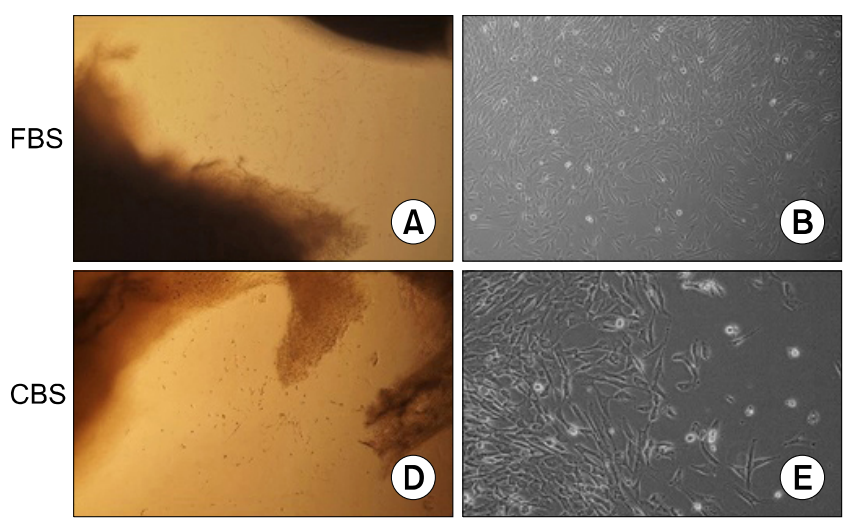

either $10 \%$ CBS or $10 \%$ FBS, were trypsinized as described previously, washed with PBS and $\left(5 \times 10^{5}\right)$ cells were suspended and stained with antibodies: CD105-PE (Invitrogen, USA), CD90-FITC (Santa Cruz Biotechnology, USA), CD44-PE (BioRad, USA), CD34-PE (BD Bioscience, USA), CD45-FITC (Invitrogen, USA), CD14-FITC (BioRad, USA). Samples were analyzed using the FACSCalibur (Becton Dickinson, USA) and DATA were analyzed with CellQuest software (Becton Dickinson, USA) and Flowing Software V2.5.1 (Turku Centre for Biotechnology, Finland).

Immunofluorescence: Cells were grown at chamber (chamber slide system, Labtek, USA) washed with PBS and fixed with $10 \%$ formaldehyde for $30 \mathrm{~min}$. Blocking was performed by incubating fixed cells in blocking solution contains $2 \%$ bovine serum albumin (BSA) (Carl Roth, Germany) and 2\% fetal bovine serum (FBS) (Sigma-Aldrich, Germany) for 1 hour. Then cells were incubated overnight at $4^{\circ} \mathrm{C}$ with antibodies against CD105-PE (Invitrogen, USA), CD90- FITC (Santa Cruz biotechnology, USA), CD44-FITC (BD Bioscience, USA). After washing, cell nuclei were counterstained with DAPI (4',6-diamidino-2-phenylindole) $1 \mu \mathrm{g} / \mathrm{ml}$ for $5 \mathrm{~min}$. Images were taken using Olympus fluorescent microscope (Olympus, Japan).

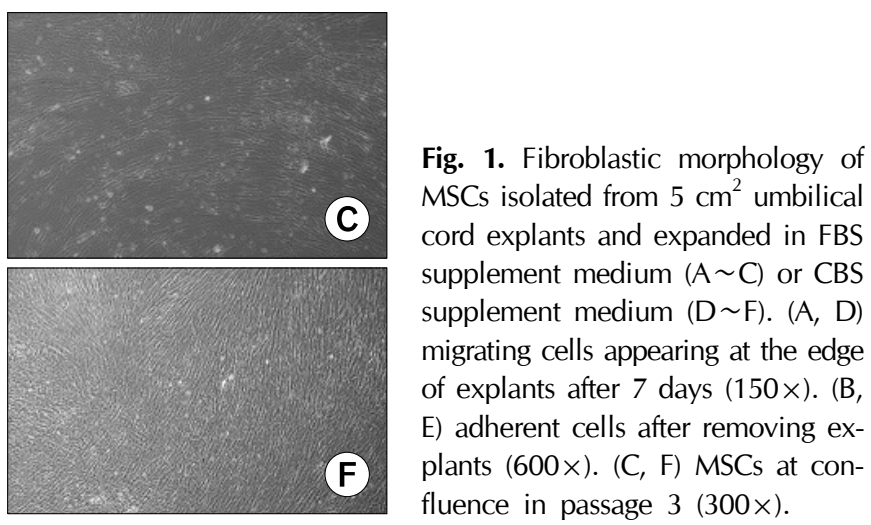

Statistical analysis: Data were analyzed using the SPSS program version 16.0 and expressed as means \pm SE. A t-test was used to determine p-values.

\section{Results}

\section{Isolation of MSCs from umbilical cord tissue}

After 7 12 days of explants culture, many cells migrated from explants, adhered to the surface of flasks, and were cultured in medium supplemented with either CBS or FBS. Explants were removed after 2 weeks and adherent cells had the ability to proliferate and expand, and were later subcultured when reaching $70 \sim 90 \%$ confluence. Adherent Cells isolated from UC tissues in medium supplemented with either $10 \%$ CBS or $10 \%$ FBS showed fibroblastic morphology (spindle- shaped) with multiple nucleoli which is a typical morphology of MSCs. Moreover, cells maintained their morphology when sub-cultured beyond passage 3 in medium supplemented with either CBS or FBS (Fig. 1).

\section{Expansion and proliferation kinetics}

Population doubling time (PDT) for isolated MSCs using medium supplemented with either $10 \%$ CBS or $10 \%$ FBS did not change significantly between two media 

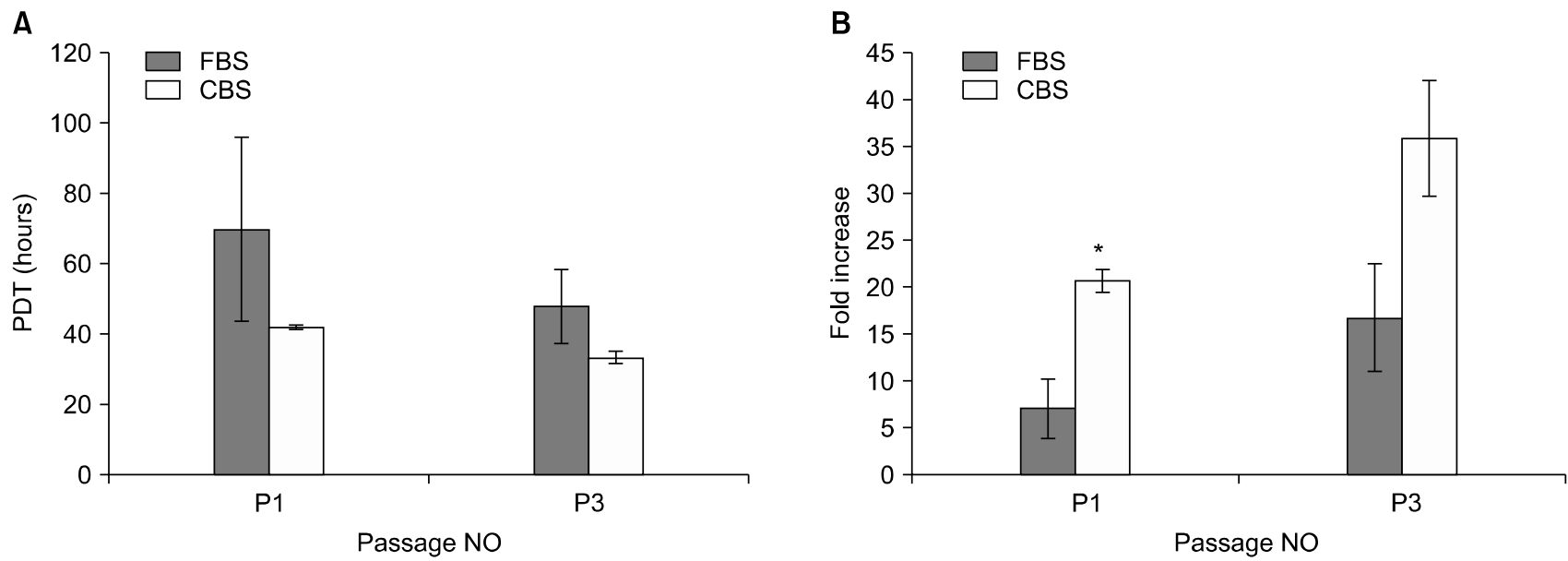

Fig. 2. Proliferation kinetics of UCMSCs in CBS and FBS supplemented media. Initial densities at each passage were 10,000/well and results are shown as means \pm SE. (A) Population doublings time (PDT). (B) Fold increase. ${ }^{*} p$-value $<0.05$.
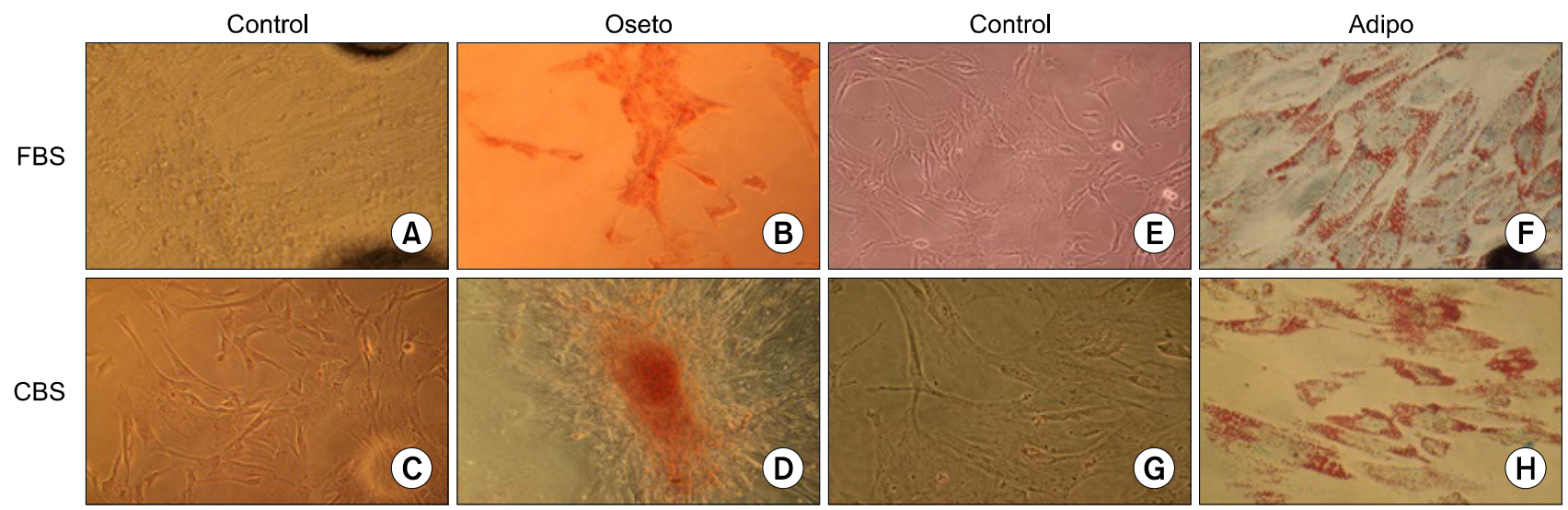

Fig. 3. Adipogenic and osteogenic differentiation of MSCs. (A, C, E, G) are controls were MSCs from passage 3 were cultured and stained similarly in the presence of FBS supplemented medium (A, E) or CBS supplemented medium (C, G). (B, D) osteogenic differentiation showed positive staining of mineralized by Alizarin red. $(F, H)$ adipogenic differentiation showed positive staining of lipid vacuoles by Sudan III.

throughout culture period from passage 1 to 3 (p-value $>$ 0.05), with lower PDT for cells cultured in medium supplement with $10 \%$ CBS than for cells cultured in medium supplement with $10 \%$ FBS. In addition, fold increase (FD) for cells cultured in medium supplement with $10 \%$ CBS was higher than for cells cultured in medium supplement with 10\% FBS throughout culture period from passage 1 to 3 (p-value $<0.05$ ), with significance appeared only in passage 1 (Fig. 2).

\section{Adipogenic and osteogenic differentiation}

MSCs cultured in medium supplement with either CBS or FBS and directed to differentiate to adipocytes showed morphology changes and displayed the accumulation of lipid vacuoles, which stained positively with sudan III (Fig. 3F, 3H). In addition, MSC cultured in osteogenic differentiated medium showed morphology changes and stained in red color when stained with alizarin red, indicating the presence of calcium deposits for cultured cells in medium supplement with either CBS or FBS (Fig. 3B, 3D).

\section{Molecular phenotyping}

RNA was extracted from MSCs in P3 cultured in (DMEM, 10\% CBS) and (DMEM, 10\% FBS), after which RT-PCR reaction was performed. RT-PCR products for MSCs specific markers including CD105, CD44, and CD90 were visualized on gel electrophoresis (Fig. 4).

\section{Immunophenotyping characterization}

Immunophenotypic profile for surface markers of expanded cells in P3 was evaluated by flow cytometry. Cells 


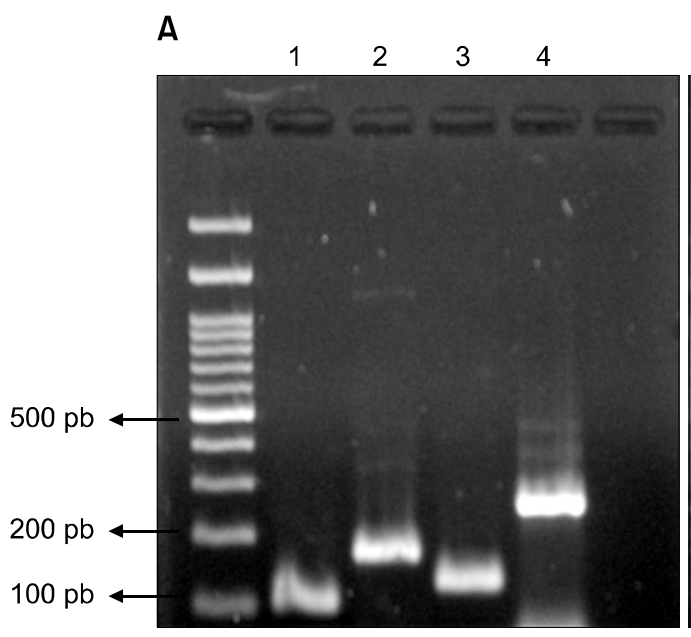

FBS
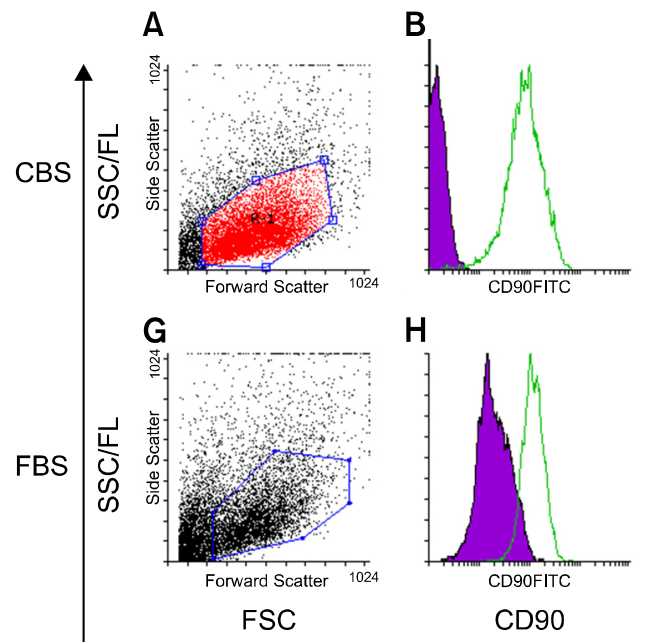

B

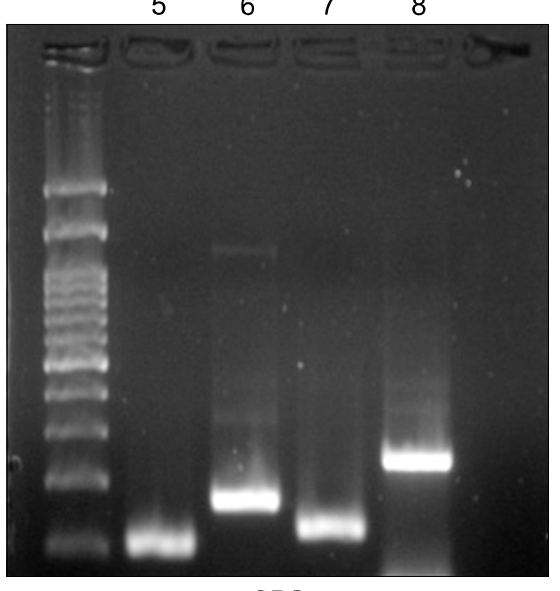

Fig. 4. $2 \%$ agarose gel electrophoresis for amplified transcripts expressed by MSCs cultured in FBS supplement medium (A) or in CBS supplement medium $(B) .(1,5)$ beta-actin amplicon of 100bp used as control. $(2,6)$ CD105 amplicon of 165 bp. (3. 7) CD90 amplicon of 124 bp. $(4,8)$ CD44 amplicon of 233 bp.
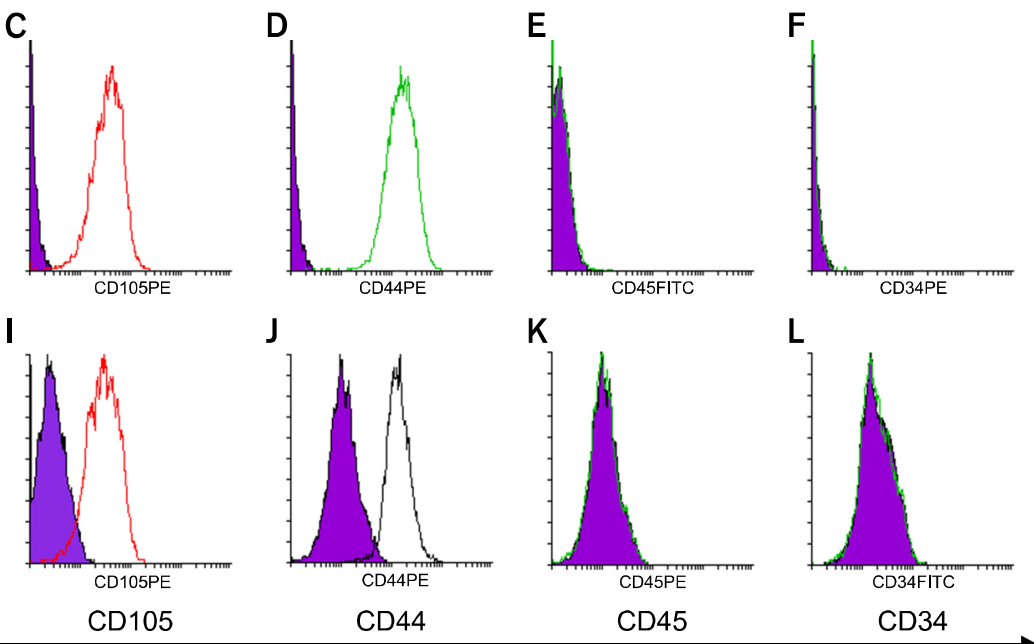

Fig. 5. Flow cytometry of UCMSCs cultured in human CBS supplemented medium $(A \sim F)$ and in FBS supplemented medium $(G \sim L)$. (A, G) FSC and SSC distribution of gated cells. Cells stained positive for CD90 (B, H), CD105 (C, I), CD44 (D, J), while stained negative for CD45 $(\mathrm{E}, \mathrm{K})$, and $\mathrm{CD} 34(\mathrm{~F}, \mathrm{~L})$.

cultured in (DMEM, 10\% CBS) and (DMEM, 10\% FBS) medium were positive for (CD90, CD105, CD44) and negative for (CD34, CD45) surface markers. Nonetheless, expression of (CD90, CD105, CD44) was considerably higher for cells cultured in CBS than those in FBS as shown in (Fig. 5).

\section{Immunofluorescence}

The expression of MSCs specific markers was also confirmed by immunofluorescence. Immunofluorescent analysis of MSCs cultured in CBS supplemented medium and FBS supplemented medium confirmed the expression of specific markers (CD105, CD44, and CD90) of MSCs cultured in either medium (Fig. 6).

\section{Discussion}

Mesenchymal stem cells are multipotent cells that were increasingly used in clinical studies (4). Two basic methods are implemented to isolate UCMSCs; explant and enzymatic methods that could differ from lab to lab (2). In addition, the majority of protocols still use FBS as supplement in culture media, which is considered the golden standard as medium supplement despite its numerous limitations (26).

In recent years, researchers have focused on optimizing conditions for isolation and expansion of MSCs for clinical trial proposes using xenogenic-free medium including serum-free medium and human product supplement me- 


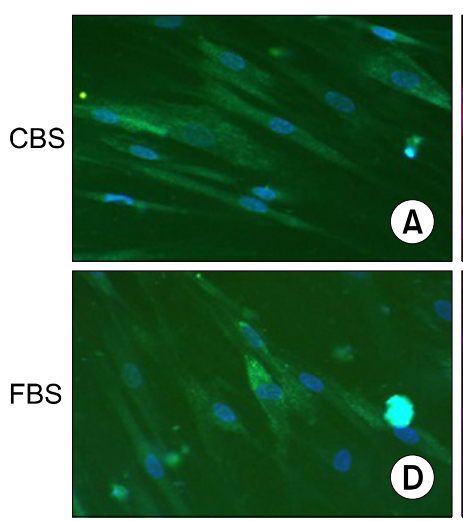

CD105

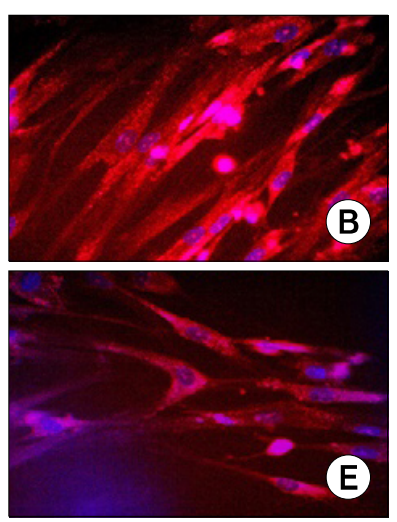

CD44

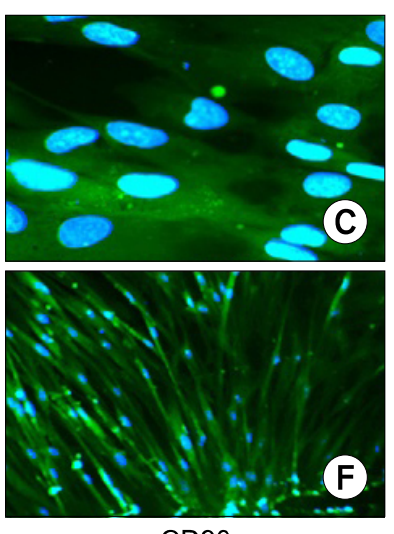

CD90
Fig. 6. Immunofluorescence for CD105, CD44 and CD90 expression in MSCs cultured in CBS supplement media $(A \sim C)$ or in FBS supplement media $(D \sim F)$. Positive staining for CD105 (A, D), CD44 (B, E), and CD90 (C, F). Nuclei were counterstained with DAPI (blue). dia (34). Platelet lysate (17, 27), adult human serum (29), human plasma (28) and human umbilical cord serum have been used as alternative supplement of fetal bovine serum (30). Many groups have studied the effects of human blood-derived supplement medium on MSCs culture showing that these media could be useful in clinical grade MSC production by decreasing scale up time $(13,27,35$, 36). On the other hand, whole pieces of umbilical cord have also been cultured in human blood-derived supplement medium $(23,37)$ and in fetal bovine serum supplement media (38). However, a few researchers have studied the effect of umbilical cord blood serum on isolating UC tissue-derived mesenchymal stem cells by the explant method. Indeed, the effect of CBS was more studied on bone marrow derived MSCs than Umbilical cord tissue derived MSCs (30, 35, 39, 40).

In our study, we tested a simple protocol to isolate UCMSCs by explant method with minimal manipulation of UC by culturing $5 \mathrm{~mm}$ pieces of umbilical cord tissue without chopping the cord pieces.

Our results indicated that MSCs with fibroblastic morphology can be isolated from $5 \mathrm{~cm}^{2}$ umbilical cord pieces by its ability to migrate and adhere to plastic surfaces when cord pieces were cultured in CBS supplement medium, similar to their morphology when using FBS supplement medium in the same conditions (Fig. 1). Our results were similar to Khushnuma et al, who used a similar protocol to isolate UCMSCs, except for adding fibroblast growth factor (FGF) to culture media supplement with umbilical cord blood serum (41). In our study, we applied CBS as supplement and used explant method without adding any growth factors and showed that MSCs are easily and cost-effectively isolated from cord tissue. This indicates that using explant method combined with cord blood serum alone could be used as an alternative to FBS, beside the advantage of CBS as a xenogenic-free medium.
CBS also showed that it enhanced self-renewal and differentiation potential of bone marrow derived MSCs (40). We also showed that UCMSCs maintained their morphology and multipotent differentiation capacity, i.e., their ability to differentiate into adipocytes and osteocytes when they were cultured in CBS as well as FBS supplement media (Fig. 2).

MSCs isolated and expanded in CBS supplemented medium up to passage 3 had considerably lower population doubling times than for cells isolated and expanded in FBS supplemented medium and narrower range; PDT for cells in CBS ranged between 30 and 48 h, while PDT for cells in FBS ranged between 38 and $95 \mathrm{~h}$ ). Moreover, MSCs cultured in CBS supplement medium had higher fold increase compared to MSCs cultured in FBS supplement medium with significant difference only in passage 1 , and no difference between the two media in passage 3 possibly due to equivalent adaption of cells to either supplement media during elongated culturing (Fig. 3). Importantly, low population doubling times and high fold increase allows getting a large cell count of MSCs suitable for clinical application in short period, i.e., in early passages.

At molecular level, MSCs are characterized by expressing CD90, CD105 and CD44 surface markers, and lacking the expression of CD34 and CD45 (18). Our data from RT-PCR, flow cytometry, and immunofluorescence collectively showed that isolated and expanded cells from umbilical cord tissue using explant method and either CBS supplement medium or FBS supplement medium corresponded well with the standard positive and negative molecular profile, indicating that explant method combined with CBS-supplement media did not alter expression of MSCs surface markers during isolation and expansion period (Figs. 4 6). Interestingly, expressing of CD90, CD105 and CD44 surface markers were considerably higher in 
CBS than FBS supplement media, possibly indicating a favored profile when culturing cells in CBS (Fig. 5).

\section{Conclusions}

Our data strongly support the plausibility of using explant method combined with pooled CBS as alternatives of enzyme digestion and FBS in the clinical setup as this method is simple and cost-effective to isolate, culture and expand UCMSCs, maintaining their stemness characteristics including self-renewal ability, multi-differentiation potential, and unique surface markers. The simplicity and easiness of this protocol makes it favorable for basic and clinical research as well as training schemes.

\section{Acknowledgments}

The main body of this work was carried out at the department of pharmaceutical biotechnology at the National Commission for Biotechnology, Damascus, Syria, with a partial support from the scientific committee at the University of Damascus and the ministry of Higher Education in Syria. The authors thank Ms. Reham Antaki, Mr Mohammad Bahjat, Ms. Ranad AlKadry, Ms. Arwa Farhat and Ms. Nour Shamsuldeen for their kind help and support.

\section{Potential conflict of interest}

The authors have no conflicting financial interest.

\section{References}

1. Weiss ML, Troyer DL. Stem cells in the umbilical cord. Stem Cell Rev 2006;2:155-162

2. Can A, Karahuseyinoglu S. Concise review: human umbilical cord stroma with regard to the source of fetus-derived stem cells. Stem Cells 2007;25:2886-2895

3. Chua SJ, Bielecki R, Wong CJ, Yamanaka N, Rogers IM, Casper RF. Neural progenitors, neurons and oligodendrocytes from human umbilical cord blood cells in a serum-free, feeder-free cell culture. Biochem Biophys Res Commun 2009;379:217-221

4. Ding DC, Chang YH, Shyu WC, Lin SZ. Human umbilical cord mesenchymal stem cells: a new era for stem cell therapy. Cell Transplant 2015;24:339-347

5. Wei X, Yang X, Han ZP, Qu FF, Shao L, Shi YF. Mesenchymal stem cells: a new trend for cell therapy. Acta Pharmacol Sin 2013;34:747-754

6. Cui R, Rekasi H, Hepner-Schefczyk M, Fessmann K, Petri RM, Bruderek K, Brandau S, Jäger M, Flohé SB. Human mesenchymal stromal/stem cells acquire immunostimulatory capacity upon cross-talk with natural killer cells and might improve the NK cell function of immunocompromised patients. Stem Cell Res Ther 2016;7:88

7. Fierabracci A, Del Fattore A, Muraca M. The immunoregulatory activity of mesenchymal stem cells: 'state of art' and 'future avenues'. Curr Med Chem 2016;23:3014-3024

8. Helal MA, Shaheen NE, Abu Zahra FA. Immunomodulatory capacity of the local mesenchymal stem cells transplantation after severe skeletal muscle injury in female rats. Immunopharmacol Immunotoxicol 2016:1-9

9. Lee OK, Kuo TK, Chen WM, Lee KD, Hsieh SL, Chen TH. Isolation of multipotent mesenchymal stem cells from umbilical cord blood. Blood 2004;103:1669-1675

10. Wolfe M, Pochampally R, Swaney W, Reger RL. Isolation and culture of bone marrow-derived human multipotent stromal cells (hMSCs). Methods Mol Biol 2008;449:3-25

11. Avola R, Graziano AC, Pannuzzo G, Cardile V. Human mesenchymal stem cells from adipose tissue differentiated into neuronal or glial phenotype express different aquaporins. Mol Neurobiol 2016 [Epub ahead of print]

12. Ou H, Zhao S, Peng Y, Xiao X, Wang Q, Liu H, Xiao X, Yang $M$. Comparison of bone marrow tissue- and adipose tissue-derived mesenchymal stem cells in the treatment of sepsis in a murine model of lipopolysaccharide-induced sepsis. Mol Med Rep 2016;14:3862-3870

13. Ding Y, Lu Z, Yuan Y, Wang X, Li D, Zeng Y. Comparison of human cord blood mesenchymal stem cell culture between using human umbilical cord plasma and using fetal bovine serum. Sheng Wu Yi Xue Gong Cheng Xue Za Zhi 2013;30:1279-1282

14. Pelekanos RA, Sardesai VS, Futrega K, Lott WB, Kuhn M, Doran MR. Isolation and expansion of mesenchymal stem/stromal cells derived from human placenta tissue. J Vis Exp 2016;(112)

15. Fei X, Jiang S, Zhang S, Li Y, Ge J, He B, Goldstein S, Ruiz G. Isolation, culture, and identification of amniotic fluid-derived mesenchymal stem cells. Cell Biochem Biophys 2013;67:689-694

16. Wouters G, Grossi S, Mesoraca A, Bizzoco D, Mobili L, Cignini P, Giorlandino C. Isolation of amniotic fluid-derived mesenchymal stem cells. J Prenat Med 2007;1:39-40

17. Pham PV, Vu NB, Pham VM, Truong NH, Pham TL, Dang LT, Nguyen TT, Bui AN, Phan NK. Good manufacturing practice-compliant isolation and culture of human umbilical cord blood-derived mesenchymal stem cells. J Transl Med 2014;12:56

18. Dominici M, Le Blanc K, Mueller I, Slaper-Cortenbach I, Marini F, Krause D, Deans R, Keating A, Prockop Dj, Horwitz E. Minimal criteria for defining multipotent mesenchymal stromal cells. The International Society for Cellular Therapy position statement. Cytotherapy 2006;8: 315-317

19. Mennan C, Wright K, Bhattacharjee A, Balain B, Richardson J, Roberts S. Isolation and characterisation of mesenchymal stem cells from different regions of the human umbilical cord. Biomed Res Int 2013 doi: 10.1155/ 2013/916136

20. Iftimia-Mander A, Hourd P, Dainty R, Thomas RJ. 
Mesenchymal stem cell isolation from human umbilical cord tissue: understanding and minimizing variability in cell yield for process optimization. Biopreserv Biobank 2013;11:291-298

21. Yoon JH, Roh EY, Shin S, Jung NH, Song EY, Chang JY, Kim BJ, Jeon HW. Comparison of explant-derived and enzymatic digestion-derived MSCs and the growth factors from Wharton's jelly. Biomed Res Int 2013 doi: 10.1155/ 2013/428726

22. Hendijani F, Sadeghi-Aliabadi H, Haghjooy Javanmard S. Comparison of human mesenchymal stem cells isolated by explant culture method from entire umbilical cord and Wharton's jelly matrix. Cell Tissue Bank 2014;15:555-565

23. Capelli C, Gotti E, Morigi M, Rota C, Weng L, Dazzi F, Spinelli O, Cazzaniga G, Trezzi R, Gianatti A, Rambaldi A, Golay J, Introna M. Minimally manipulated whole human umbilical cord is a rich source of clinical-grade human mesenchymal stromal cells expanded in human platelet lysate. Cytotherapy 2011;13:786-801

24. Hendijani F. Explant culture: An advantageous method for isolation of mesenchymal stem cells from human tissues. Cell Prolif 2017;50

25. Wolfe M, Tucker A, Reger RL, Prockop DJ. Multipotent stromal cells (hMSCs). In: Masters JRW, Palsson B, editor. Human Adult Stem Cells. Dordrecht, Netherlands: Springer; 2009. $45-72$

26. Tekkatte C, Gunasingh GP, Cherian KM, Sankaranarayanan K. "Humanized" stem cell culture techniques: the animal serum controversy. Stem Cells Int 2011 doi: 10.4061/2011/ 504723

27. Bieback K. Platelet lysate as replacement for fetal bovine serum in mesenchymal stromal cell cultures. Transfus Med Hemother 2013;40:326-335

28. Díez JM, Bauman E, Gajardo R, Jorquera JI. Culture of human mesenchymal stem cells using a candidate pharmaceutical grade xeno-free cell culture supplement derived from industrial human plasma pools. Stem Cell Res Ther 2015;6:28

29. Tateishi K, Ando W, Higuchi C, Hart DA, Hashimoto J, Nakata K, Yoshikawa H, Nakamura N. Comparison of human serum with fetal bovine serum for expansion and differentiation of human synovial MSC: potential feasibility for clinical applications. Cell Transplant 2008;17:549-557

30. Shetty P, Bharucha K, Tanavde V. Human umbilical cord blood serum can replace fetal bovine serum in the culture of mesenchymal stem cells. Cell Biol Int 2007;31:293-298

31. Salzig D, Leber J, Merkewitz K, Lange MC, Köster N,
Czermak P. Attachment, growth, and detachment of human mesenchymal stem cells in a chemically defined medium. Stem Cells Int 2016 doi: 10.1155/2016/5246584

32. Kinzebach S, Bieback K. Expansion of mesenchymal stem/stromal cells under xenogenic-free culture conditions. In: Weyand B, Benda C, editor. Mesenchymal stem cells: basics and clinical application. Heidelberg, Berlin: Springer; 2013. $33-57$

33. Song HJ, Zhang P, Guo XJ, Liao LM, Zhou ZM, Sha JH, Cui YG, Ji H, Liu JY. The proteomic analysis of human neonatal umbilical cord serum by mass spectrometry. Acta Pharmacol Sin 2009;30:1550-1558

34. Schnitzler AC, Verma A, Kehoe DE, Jing D, Murrell JR, Der KA, Aysola M, Rapiejko PJ. Bioprocessing of human mesenchymal stem/stromal cells for therapeutic use: Current technologies and challenges. Biochemical Engineering Journal 2016;108:3-13

35. Phadnis SM, Joglekar MV, Venkateshan V, Ghaskadbi SM, Hardikar AA, Bhonde RR. Human umbilical cord blood serum promotes growth, proliferation, as well as differentiation of human bone marrow-derived progenitor cells. In Vitro Cell Dev Biol Anim 2006;42:283-286

36. Hemeda H, Giebel B, Wagner W. Evaluation of human platelet lysate versus fetal bovine serum for culture of mesenchymal stromal cells. Cytotherapy 2014;16:170-180

37. Van Pham P, Truong NC, Le PT, Tran TD, Vu NB, Bui $\mathrm{KH}$, Phan NK. Isolation and proliferation of umbilical cord tissue derived mesenchymal stem cells for clinical applications. Cell Tissue Bank 2016;17:289-302

38. Shetty P, Cooper K, Viswanathan C. Comparison of proliferative and multilineage differentiation potentials of cord matrix, cord blood, and bone marrow mesenchymal stem cells. Asian J Transfus Sci 2010;4:14-24

39. Turnovcova K, Ruzickova K, Vanecek V, Sykova E, Jendelova P. Properties and growth of human bone marrow mesenchymal stromal cells cultivated in different media. Cytotherapy 2009;11:874-885

40. Tekkatte C, Vidyasekar P, Kapadia NK, Verma RS. Enhancement of adipogenic and osteogenic differentiation of human bone-marrow-derived mesenchymal stem cells by supplementation with umbilical cord blood serum. Cell Tissue Res 2012;347:383-395

41. Cooper K, SenMajumdar A, Viswanathan C. Derivation, expansion and characterization of clinical grade mesenchymal stem cells from umbilical cord matrix using cord blood serum. Int J Stem Cells 2010;3:119-128 